\section{SCIENCE CHINA \\ Physics, Mechanics \& Astronomy}

\section{-Editorial•}

Editor's Focus
July 2019 Vol. 62 No. 7: 974220

https://doi.org/10.1007/s11433-019-9378-y

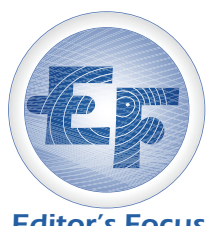

\title{
Perovskite solar cells fly in the sky
}

Near space has become the focus of attention and competition among the world's major powers. In order to utilize near space and expand human activity in space, many countries have developed near-space aircrafts. In addition, near space contains abundant physical phenomena to be detected and scientific laws to be discovered. The scientific exploration of the near space will unveil its mystery for human beings.

In this research field, energy technology determines the steady operation of the aircraft. As a recent emerging photovoltaic technology, perovskite solar cells (PSCs) exhibit outstanding efficiency, high power-per-weight, and excellent radiation resistance. Therefore, PSCs are considered to be the new-generation energy technology for near-space application.

Recently, a group of researchers at Peking University in China, led by Dr. Rui Zhu and Prof. Qihuang Gong in collaboration with Prof. Guoning Xu from Academy of Opto-Electronics, CAS, and Prof. Wei Huang from Northwestern Polytechnical University, have reported the stability study of PSCs in near space [1]. Researchers demonstrated the attempt for the stability study of large-area PSCs (active area of $1.00 \mathrm{~cm}^{2}$ ) in near space. The devices were fixed on the high-altitude balloon risen from ground to near space at an altitude of $35 \mathrm{~km}$ in Inner Mongolian Area, China. The results showed that the device based on $\mathrm{FA}_{0.81} \mathrm{MA}_{0.10} \mathrm{Cs}_{0.04} \mathrm{PbI}_{2.55} \mathrm{Br}_{0.40}$ retained $95.19 \%$ of its initial power conversion efficiency during the test under AM0 illumination.

Researchers also expect that the stability research of PSCs in near space conditions will be helpful to extend the application of PSCs. This work also opens the route for PSCs in future space application.

XinCheng Xie Editor-in-Chief

School of Physics, Peking University, Beijing 100871, China

1 Y. G. Tu, G. N. Xu, X. Y. Yang, Y. F. Zhang, Z. J. Li, R. Su, D. Y. Luo, W. Q. Yang, Y. Miao, R. Cai, L. H. Jiang, X. W. Du, Y. C. Yang, Q. S.
Liu, Y. Gao, S. Zhao, W. Huang, Q. H. Gong, and R. Zhu, Sci. ChinaPhys. Mech. Astron. 62, 974221 (2019). 